\title{
The Uniqueness Theorem for the Heat Equation on the Heisenberg Group
}

\author{
Yasuyuki OKA \\ National Institute of Technology, Kushiro College \\ (Communicated by K. Yoshitomi)
}

\begin{abstract}
The aim of this paper is to establish the uniqueness theorem for the Cauchy problem for the heat equation with the Tikhonov condition on the Heisenberg group. To do this, we give Green's formula and show the existence of a Lipschitz cut-off function on the Heisenberg group in accordance with the idea in [7].
\end{abstract}

\section{Introduction}

It is known that the Cauchy problem for the heat equation

$$
\left\{\begin{array}{l}
\frac{\partial u_{t}(x)}{\partial t}=\Delta u_{t}(x), \text { in } \mathbb{R}^{d} \times(0, T) \\
\left.u_{t}(x)\right|_{t=0}=u_{0}(x)
\end{array}\right.
$$

for any continuous function $u_{0}$ has at most one bounded solution. Furthermore, if $u_{t}(x)$ solves the Cauchy problem with an initial value $u_{0}=0$ and satisfies the condition

$$
\left|u_{t}(x)\right| \leq C e^{C|x|^{2}}
$$

for some constant $C$ and all $t>0, x \in \mathbb{R}^{d}$, then $u \equiv 0$. This is called the Tikhonov theorem on the Euclidean space (see [17]).

On the other hand, the Tikhonov theorem on a Riemannian manifold was given in [6] and [7] with a complete proof. Moreover this theorem on the Carnot groups having the Heisenberg group as a typical example was given in [9] with a comment that one can obtain exactly the same proof in [7] by existence of Lipschitz cut-off function and integration by part.

Accordingly, we focus on the Heisenberg group and give a complete proof of the Tikhonov theorem for the Cauchy problem of the heat equation on the Heisenberg group,

$$
\left\{\begin{array}{l}
\frac{\partial U_{s}(g)}{\partial s}=\Delta_{\mathbb{H}^{d}} U_{s}(g), \text { in } \mathbb{H}^{d} \times(0, S) \\
\left.U_{s}(g)\right|_{s=0}=U_{0}(g)
\end{array}\right.
$$

Received March 23, 2015; revised May 11, 2016

Mathematics Subject Classification: 46F05, 46F15, 81S30

Key words and phrases: a uniqueness of the Cauchy problem on the Heisenberg group, heat equation 
following the proof in [7].

The Heisenberg group is also the simplest and typical example of the sub-Riemannian manifold. Moreover it is a step 2 sub-Riemannian manifold. The sub-Riemannian manifold may be interpreted as a generalization of the Riemannian manifold. The difference is that the motion for the sub-Riemannian manifold is restricted to the horizontal direction (for the Riemannian manifold, we can measure the velocity and distance in all directions). Since it is difficult to consider the sub-Riemannian manifolds, we only consider the Heisenberg group case which has been investigated by many mathematicians.

Recently, we have studied the heat kernel method on the Heisenberg group (see [13] and [14]). The heat kernel method, introduced in [11] by T. Matsuzawa, is the method to characterize the generalized functions on the Euclidean space by the initial value of the solutions of the heat equation. The Tikhonov theorem on the Heisenberg group plays an important role to construct the heat kernel method on the Heisenberg group.

The plan of this paper is as follows: In section 2, we will recall the definition and the properties of the Heisenberg group and give the sub-Riemannian metric on the Heisenberg group. In section 3, we consider the Lipschitz functions and the gradient in the Heisenberg group. We also give Green's formula on the Heisenberg group. Finally, in section 4, we will give the statement and the proof of the our main theorem.

\section{The Heisenberg group $\mathbb{H}^{d}$}

First of all, we fix some notations. We use a multi-index $\alpha \in \mathbb{Z}_{+}^{d}$, namely, $\alpha=$ $\left(\alpha_{1}, \ldots, \alpha_{d}\right)$, where $\alpha_{i} \in \mathbb{Z}$ and $\alpha_{i} \geq 0$. So, for $x \in \mathbb{R}^{d}, x^{\alpha}=x_{1}^{\alpha_{1}} \cdots x_{d}^{\alpha_{d}}$ and $\partial_{x}^{\alpha}=\partial_{x_{1}}^{\alpha_{1}} \cdots \partial_{x_{d}}^{\alpha_{d}}$, where $\partial_{x_{j}}^{\alpha_{j}}=\left(\partial / \partial x_{j}\right)^{\alpha_{j}}$. Moreover $\Delta=\sum_{j=1}^{d} \partial^{2} / \partial x_{j}^{2}$.

We recall the definition and the properties of the Heisenberg group. We refer to [1], [2], [4], [8], [15] and [16]. Let $g=(x, y, t)$ and $g^{\prime}=\left(x^{\prime}, y^{\prime}, t^{\prime}\right) \in \mathbb{R}^{d} \times \mathbb{R}^{d} \times \mathbb{R}=\mathbb{R}^{2 d+1}$. Then we define the group law of $\mathbb{R}^{2 d+1}$ by

$$
(x, y, t)\left(x^{\prime}, y^{\prime}, t^{\prime}\right)=\left(x+x^{\prime}, y+y^{\prime}, t+t^{\prime}+2\left(x^{\prime} \cdot y-x \cdot y^{\prime}\right)\right),
$$

where $x \cdot y=\sum_{j=1}^{d} x_{j} y_{j}$. The group $\mathbb{R}^{2 d+1}$ with respect to the group law defined by (1) is called the Heisenberg group and denoted by $\mathbb{H}^{d}$. Its identity element is $\boldsymbol{e}=(0,0,0)$ and the inverse of the element $(x, y, t)$ is $(x, y, t)^{-1}=(-x,-y,-t)$. The Heisenberg group $\mathbb{H}^{d}$ is a locally compact Hausdorff group and its Haar measure is the Lebesgue measure $d x d y d t$. The left-invariant vector fields in the Heisenberg group $\mathbb{H}^{d}$ as $\mathbb{R}^{2 d+1}$ are represented by

$$
X_{j}=\frac{\partial}{\partial x_{j}}+2 y_{j} \frac{\partial}{\partial t}, \quad X_{d+j}=\frac{\partial}{\partial y_{j}}-2 x_{j} \frac{\partial}{\partial t} \text { and } X_{2 d+1}=\frac{\partial}{\partial t}
$$

for $j=1,2, \ldots, d$ and these make a basis for the Lie algebra of $\mathbb{H}^{d}$. Since their first brackets

$$
\left[X_{j}, X_{d+j}\right]=-4 X_{2 d+1},
$$


the induced geometry is step 2 . The sub-Laplacian $\Delta_{\mathbb{H}^{d}}$ on $\mathbb{H}^{d}$ is defined by

$$
\Delta_{\mathbb{H} d}=\sum_{j=1}^{2 d} X_{j}^{2} .
$$

We consider the heat operator

$$
\frac{\partial}{\partial s}-\Delta_{\mathbb{H}^{d}}
$$

on $\mathbb{H}^{d} \times(0, \infty)$.

Let $\lambda>0$. Then we define the dilations $\delta_{\lambda}$ by

$$
\delta_{\lambda}(x, y, t)=\left(\lambda x, \lambda y, \lambda^{2} t\right)
$$

for $(x, y, t) \in \mathbb{H}^{d}$. The homogeneous dimension $Q$ of $\mathbb{H}^{d}$ is given by $Q=2 d+2$. Moreover, a function $u$ from $\mathbb{H}^{d}$ to $\mathbb{C}$ is called the Heisenberg-homogeneous of degree $k \in \mathbb{Z}$ if $u \circ$ $\delta_{\lambda}=\lambda^{k} u$ for $\lambda>0$. Especially the Heisenberg-homogeneous of degree of the distance function $\rho$ defined by $\rho(g)=\left(\left(x^{2}+y^{2}\right)^{2}+t^{2}\right)^{\frac{1}{4}}$ for $g=(x, y, t) \in \mathbb{H}^{d}$ is one, that is, $\rho\left(\lambda x, \lambda y, \lambda^{2} t\right)=\lambda \rho(x, y, t)$. The following estimate also holds:

$$
\rho\left(g^{\prime-1} g\right) \leq \rho(g)+\rho\left(g^{\prime}\right) .
$$

The distance between two points $g$ and $g^{\prime}$ in $\mathbb{H}^{d}$ is given by $d_{K}\left(g, g^{\prime}\right):=\rho\left(g^{-1} g\right)$. Especially, we denote by $d_{K}(g)$ the distance from the origin. This distance function $\rho$ is called Korányi norm and the distance $d_{K}$ is called Korányi distance.

The horizontal distribution is defined by $\mathcal{H}_{g}=\operatorname{span}_{g}\left\{X_{1}, X_{2}, \ldots, X_{2 d}\right\}$. Then we shall consider the non-degenerate, positive definite bilinear form $\langle\cdot, \cdot\rangle: \mathcal{H}_{g} \times \mathcal{H}_{g} \rightarrow \mathbb{R}$ at any point $g \in \mathbb{H}^{d}$ such that $\left\langle X_{i}, X_{j}\right\rangle=\delta_{i, j}(i, j=1,2, \ldots, 2 d)$, where $\delta_{i, j}$ means Kronecker's delta. The length $l(\gamma)$ of the horizontal curve $\gamma(t), t \in[a, b]$ is defined by

$$
l(\gamma)=\int_{a}^{b}\langle\dot{\gamma}(t), \dot{\gamma}(t)\rangle d t .
$$

The Carnot-Carathéodry distance $d_{C C}\left(g, g^{\prime}\right)$ between two points $g, g^{\prime} \in \mathbb{H}^{d}$ is defined by the infimum of the lengths of all smooth horizontal curves joining $g$ to $g^{\prime}$ (see [1]). These distances are bi-Lipschitz equivalent. Thus, there exists a constant $C_{1}>1$ such that

$$
\frac{1}{C_{1}} d_{C C} \leq d_{K} \leq C_{1} d_{C C}
$$

(for instance, see [10]). 


\section{The Lipschitz functions and the gradient $\tilde{\nabla}$ in $\mathbb{H}^{d}$}

Let $f$ be a function on $\mathbb{H}^{d}$. Then we say that $f$ is $L$-Lipschitz on $\mathbb{H}^{d}$ if there exists a constant $L$ such that

$$
\left|f(g)-f\left(g^{\prime}\right)\right| \leq \operatorname{Ld}_{C C}\left(g, g^{\prime}\right)
$$

for any $g, g^{\prime} \in \mathbb{H}^{d}$. The constant $L$ is a called the Lipschitz constant of $f$. The set of all Lipschitz functions on $\mathbb{H}^{d}$ is denoted by $\operatorname{Lip}\left(\mathbb{H}^{d}\right)$. We define the Lipschitz semi norm $\|f\|_{L i p\left(\mathbb{H}^{d}\right)}$ of $f$ by

$$
\|f\|_{L i p\left(\mathbb{H}^{d}\right)}=\sup _{g, g^{\prime} \in \mathbb{H}^{d}, g \neq g^{\prime}} \frac{\left|f(g)-f\left(g^{\prime}\right)\right|}{d_{C C}\left(g, g^{\prime}\right)} .
$$

From the definition, we can see that $\operatorname{Lip}\left(\mathbb{H}^{d}\right) \subset C\left(\mathbb{H}^{d}\right)$ immediately.

Let the function $\eta \mapsto \eta_{+}$be defined by

$$
\eta_{+}= \begin{cases}\eta, & \eta>0 \\ 0, & \eta \leq 0\end{cases}
$$

for $\eta \in \mathbb{R}$.

Proposition 1. Let $d_{C C}(g, E)=\inf _{z \in E} d_{C C}(g, z)$ for any non-empty set $E \subset \mathbb{H}^{d}$ and any point $g \in \mathbb{H}^{d}$. Then the function $g \mapsto\left(d_{C C}(g, E)-R\right)_{+}$for some $R>0$ is 1-Lipschitz function on $\mathbb{H}^{d}$.

Proof. We prove Proposition 1 dividing it in two cases, (i) case $d_{C C}(g, E)>R$ and $d_{C C}\left(g^{\prime}, E\right) \leq R\left(d_{C C}(g, E) \leq R\right.$ and $d_{C C}\left(g^{\prime}, E\right)>R$ ), (ii) $d_{C C}(g, E)>R$ and $d_{C C}\left(g^{\prime}, E\right)>R$. Assume that $d_{C C}(g, E)>R$ and $d_{C C}\left(g^{\prime}, E\right) \leq R$. Then we can see that

$$
\left(d_{C C}(g, E)-R\right)_{+}-\left(d_{C C}\left(g^{\prime}, E\right)-R\right)_{+}=d_{C C}(g, E)-R \leq d_{C C}\left(g, g^{\prime}\right) .
$$

Next we shall consider the case (ii) $d_{C C}(g, E)>R$ and $d_{C C}\left(g^{\prime}, E\right)>R$. For any $\varepsilon>0$, there exists $z \in E$ such that

$$
d_{C C}\left(g^{\prime}, E\right) \geq d_{C C}\left(g^{\prime}, z\right)-\varepsilon .
$$

Thus, by using the triangle inequality, we obtain

$$
\begin{aligned}
\left(d_{C C}(g, E)-R\right)_{+}-\left(d_{C C}\left(g^{\prime}, E\right)-R\right)_{+} & =d_{C C}(g, E)-d_{C C}\left(g^{\prime}, E\right) \\
& \leq d_{C C}(g, z)-\left(d_{C C}\left(g^{\prime}, z\right)-\varepsilon\right) \\
& =d_{C C}(g, z)-d_{C C}\left(g^{\prime}, z\right)+\varepsilon \\
& \leq d_{C C}\left(g, g^{\prime}\right)+\varepsilon
\end{aligned}
$$

for any $\varepsilon>0$. Therefore for any two point $g, g^{\prime} \in \mathbb{H}^{d}$, we have

$$
\left(d_{C C}(g, E)-R\right)_{+}-\left(d_{C C}\left(g^{\prime}, E\right)-R\right)_{+} \leq d_{C C}\left(g, g^{\prime}\right) .
$$


This completes the proof of Proposition 1.

Let $d_{C C}(g)=d_{C C}(g, \boldsymbol{e})$. Then from Proposition 1, we can see that the function $\left(d_{C C}(g)-R\right)_{+}$is 1-Lipschitz function. On the other hand, let the gradient $\tilde{\nabla}_{\mathbb{H}^{d}}$ on $\mathbb{H}^{d}$ be defined by $\tilde{\nabla}_{\mathbb{H}^{d}}=\left(X_{1}, \ldots, X_{d}, X_{d+1}, \ldots, X_{2 d}\right)$.

The following Proposition 2 is known (see [3], [5] and [12]):

Proposition 2. For any L-Lipschitz function $f$ on $\mathbb{H}^{d}$, the value $X_{j} f(g)$ exists for almost all $g \in \mathbb{H}^{d}, j=1,2, \ldots, 2 d$, and

$$
|X f|=\left(\sum_{j=1}^{2 d}\left(X_{j} f(g)\right)^{2}\right)^{\frac{1}{2}} \leq \text { La.e. }
$$

This Proposition 2 implies the following Proposition 3:

PROPOSITION 3. We have the following estimate:

$$
\left|\tilde{\nabla}_{\mathbb{H}^{d}}\left(d_{C C}(g)-R\right)_{+}\right| \leq 1 .
$$

As a remark, in [12], R. Monti also obtain the following result

$$
\left|\tilde{\nabla}_{\mathbb{H}^{d}} d_{C C}(g)\right|=1
$$

for all $g=(x, y, t) \in \mathbb{H}^{d}$ such that $(x, y) \neq(0,0)$.

By an easy calculation, we have the following product rule and the chain rule of the gradient $\tilde{\nabla}_{\mathbb{H}^{d}}$ for the $C^{\infty}$ functions on $\mathbb{H}^{d}$ :

$$
\tilde{\nabla}_{\mathbb{H}^{d}}(f g)=f \tilde{\nabla}_{\mathbb{H}^{d}} g+g \tilde{\nabla}_{\mathbb{H}^{d}} f, \tilde{\nabla}_{\mathbb{H}^{d}} \psi(f)=\psi^{\prime}(f) \tilde{\nabla}_{\mathbb{H}^{d}} f
$$

for $\psi \in C^{1}(\mathbb{R})$. Moreover we can express that

$$
\tilde{\nabla}_{\mathbb{H}^{d}} f=\sum_{i=1}^{2 d} X_{i}(f) X_{i}
$$

for $f \in C_{0}^{\infty}\left(\mathbb{H}^{d}\right)$. By an integration by parts, we can obtain

$$
\int_{\mathbb{H}^{d}} X_{i}(f) X_{i}(h) d g=-\int_{\mathbb{H}^{d}} f X_{i}^{2}(h) d g
$$

for $f, h \in C_{0}^{\infty}\left(\mathbb{H}^{d}\right)$.

By (4), we have for $f, h \in C_{0}^{\infty}\left(\mathbb{H}^{d}\right)$,

$$
\int_{\mathbb{H}^{d}}\left\langle\tilde{\nabla}_{\mathbb{H}^{d}} f, \tilde{\nabla}_{\mathbb{H}^{d}} h\right\rangle d g=\int_{\mathbb{H}^{d}}\left\langle\sum_{i=1}^{2 d} X_{i}(f) X_{i}, \sum_{j=1}^{2 d} X_{j}(h) X_{j}\right\rangle d g
$$




$$
\begin{aligned}
& =\sum_{i=1}^{2 d} \int_{\mathbb{H}^{d}} X_{i}(f) X_{i}(h) d g \\
& =-\sum_{i=1}^{2 d} \int_{\mathbb{H}^{d}} f X_{i}^{2}(h) d g \\
& =-\int_{\mathbb{H}^{d}} f \Delta_{\mathbb{H}^{d}} h d g .
\end{aligned}
$$

Proposition 4. Let $f, h \in C_{0}^{\infty}\left(\mathbb{H}^{d}\right)$. Then we have the following equality:

$$
\int_{\mathbb{H}^{d}} f \Delta_{\mathbb{H}^{d}} h d g=-\int_{\mathbb{H}^{d}}\left\langle\tilde{\nabla}_{\mathbb{H}^{d}} f, \tilde{\nabla}_{\mathbb{H}^{d}} h\right\rangle d g .
$$

\section{The uniqueness theorem for the heat equation on $\mathbb{H}^{d}$}

We will show the following uniqueness of the solution to the heat equation on $\mathbb{H}^{d}$.

THEOREM 1. Let $0<S \leq \infty$ be fixed and $U_{s}(g)$ be a solution to the Cauchy problem

$$
\left\{\begin{array}{l}
\frac{\partial}{\partial s} U_{s}(g)=\Delta_{\mathbb{H}^{d}} U_{s}(g), \\
U_{0}(g)=0
\end{array}\right.
$$

in $\mathbb{H}^{d} \times(0, S)$ and be a continuous function in $\mathbb{H}^{d} \times[0, S]$. Assume that for some $g_{0} \in \mathbb{H}^{d}$ and for any $R>0$, we have the following estimate:

$$
\int_{0}^{S} \int_{B\left(g_{0}, R\right)} U_{s}^{2}(g) d g d s \leq e^{f(R)},
$$

where $f(r)$ is a positive increasing function on $(0,+\infty)$ such that

$$
\int^{\infty} \frac{r}{f(r)} d r=\infty .
$$

Then $U \equiv 0$ in $\mathbb{H}^{d} \times(0, S)$.

By using the distance function $a d_{C C}(g)^{2}$ for some constant $a>0$ as $f$, Theorem 1 immediately implies the following Corollary 1 :

Corollary 1. Let $U_{s}(g)$ be a solution to the Cauchy problem (5) in $\mathbb{H}^{d} \times(0, S)$ and be a continuous function in $\mathbb{H}^{d} \times[0, S]$ satisfying the condition: There exists a constant $C>0$ such that

$$
\left|U_{s}(g)\right| \leq C e^{a d_{C C}(g)^{2}}, \quad(g, s) \in \mathbb{H}^{d} \times(0, S)
$$

for some constant $a>0$. Then $U \equiv 0$. 
By (3), we can rewrite the condition (6) in Corollary 1 by using the Korányi distance $d_{K}$ as follows: There exists a constant $C>0$ such that

$$
\left|U_{S}(g)\right| \leq C e^{a d_{K}(g)^{2}}
$$

for some constant $a>0$.

Let $B_{R}=B\left(g_{0}, R\right)=\left\{g \in \mathbb{H}^{d} \mid d_{C C}\left(g, g_{0}\right) \leq R\right\}$. Then to prove Theorem 1, we give the following Lemma 1 .

LEMMA 1. Let $U_{s}(g)$ be the solution of the heat equation in $\mathbb{H}^{d} \times(a, b)$. Assume that $U_{s}(g)$ extends to a continuous function in $\mathbb{H}^{d} \times[a, b]$. Assume also that, for any $R>0$,

$$
\int_{a}^{b} \int_{B_{R}} U_{s}^{2}(g) d g d s \leq e^{f(R)},
$$

where $f$ is a positive increasing function on $(0,+\infty)$ such that

$$
\int^{\infty} \frac{r}{f(r)} d r=\infty
$$

Then for any $R>0$ satisfying the condition

$$
b-a \leq \frac{R^{2}}{8 f(4 R)},
$$

we have the following inequality:

$$
\int_{B_{R}} U_{b}^{2}(g) d g \leq \int_{B_{4 R}} U_{a}^{2}(g) d g+\frac{4}{R^{2}} .
$$

By using this Lemma, Theorem 1 can be proved by the same method as the proof of Theorem 11. 9 in [7]. Therefore we will only show the proof of Lemma 1.

The proof of Lemma 1. Let $\sigma(g)$ be a Lipschitz function on $\mathbb{H}^{d}$ with the Lipschitz constant 1 . Then, fix a real $l \notin[a, b]$ and put

$$
\xi(g, s)=-\frac{\sigma^{2}(g)}{4(l-s)} \text { on } \mathbb{H}^{d} \times[a, b] .
$$

Then we have

$$
\left|\tilde{\nabla}_{\mathbb{H}^{d}} \xi(g, s)\right| \leq \frac{\sigma(g)}{2(l-s)}
$$

Since

$$
\frac{\partial \xi}{\partial s}=-\frac{\sigma^{2}(g)}{4(l-s)^{2}},
$$


we obtain

$$
\frac{\partial \xi}{\partial s}+\left|\tilde{\nabla}_{\mathbb{H}^{d}} \xi\right|^{2} \leq 0
$$

Now we define a function $\chi(g)$ by

$$
\chi(g)=\min \left\{\left(3-\frac{d_{C C}\left(g, g_{0}\right)}{R}\right)_{+}, 1\right\}
$$

for a given $R>0$ and $g_{0} \in \mathbb{H}^{d}$. Then we can see that the function $\chi$ satisfies $0 \leq \chi \leq 1$ on $\mathbb{H}^{d}, \chi \equiv 1$ in $B_{2 R}$ and $\chi \equiv 0$ outside $B_{3 R}$. Since the function $d_{C C}\left(g, g_{0}\right)$ is the 1-Lipschitz function, we can see that $\chi$ is the $1 / R$-Lipschitz function. By Proposition 2, we obtain

$$
\left|\tilde{\nabla}_{\mathbb{H}^{d}} \chi\right| \leq 1 / R
$$

Since all the balls in $\mathbb{H}^{d}$ are relatively compact sets, the function $\chi(g)$ has a compact support. Hence for any fixed $s \in[a, b]$, the function $U \chi^{2} e^{\xi}$ of $g$ also has a compact support.

Now multiplying the heat equation

$$
\frac{\partial U}{\partial s}=\Delta_{\mathbb{H} d} U
$$

by the function $U \chi^{2} e^{\xi}$ and integrating it over $\mathbb{H}^{d} \times[a, b]$, we have

$$
\int_{a}^{b} \int_{\mathbb{H}^{d}} \frac{\partial U}{\partial s} U \chi^{2} e^{\xi} d g d s=\int_{a}^{b} \int_{\mathbb{H}^{d}}\left(\Delta_{\mathbb{H}^{d}} U\right) U \chi^{2} e^{\xi} d g d s
$$

The integral with respect to the variable $s$ of the left hand side in (9) becomes

$$
\int_{a}^{b} \frac{\partial U}{\partial s} U \chi^{2} e^{\xi} d s=\frac{1}{2} \int_{a}^{b} \frac{\partial\left(U^{2}\right)}{\partial s} \chi^{2} e^{\xi} d s=\frac{1}{2}\left[U^{2} \chi^{2} e^{\xi}\right]_{a}^{b}-\frac{1}{2} \int_{a}^{b} \frac{\partial \xi}{\partial s} U^{2} \chi^{2} e^{\xi} d s .
$$

Since

$$
p q \leq \frac{1}{4} p^{2}+q^{2}
$$

for $p>0$ and $q>0$, by the product rule, the chain rule and the Schwarz inequality, we have

$$
\begin{aligned}
-\left\langle\tilde{\nabla}_{\mathbb{H}^{d}} U, \tilde{\nabla}_{\mathbb{H}^{d}}\left(U \chi^{2} e^{\xi}\right)\right\rangle & =-\left\langle\tilde{\nabla}_{\mathbb{H}^{d}} U,\left(\tilde{\nabla}_{\mathbb{H}^{d}} U\right) \chi^{2} e^{\xi}+U\left(\tilde{\nabla}_{\mathbb{H}^{d}} \chi^{2} e^{\xi}\right)\right\rangle \\
& \leq-\chi^{2} e^{\xi}\left|\tilde{\nabla}_{\mathbb{H}^{d}} U\right|^{2}+|U|\left|\tilde{\nabla}_{\mathbb{H}^{d}} U\right|\left|\tilde{\nabla}_{\mathbb{H}^{d}} \xi\right| \chi^{2} e^{\xi} \\
& +2\left|\tilde{\nabla}_{\mathbb{H}^{d}} U\right||\chi| \tilde{\nabla}_{\mathbb{H}^{d}} \chi|| U \mid e^{\xi} \\
& \leq-\chi^{2} e^{\xi}\left|\tilde{\nabla}_{\mathbb{H}^{d}} U\right|^{2}+|U|\left|\tilde{\nabla}_{\mathbb{H}^{d}} U\right|\left|\tilde{\nabla}_{\mathbb{H}^{d}} \xi\right| \chi^{2} e^{\xi} \\
& +\frac{1}{2}\left|\tilde{\nabla}_{\mathbb{H}^{d}} U\right|^{2} \chi^{2} e^{\xi}+2\left|\tilde{\nabla}_{\mathbb{H}^{d}} \chi\right|^{2}|U|^{2} e^{\xi} \\
& =\left(-\frac{1}{2}\left|\tilde{\nabla}_{\mathbb{H}^{d}} U\right|^{2}+\left|\tilde{\nabla}_{\mathbb{H}^{d}} U\right|\left|\tilde{\nabla}_{\mathbb{H}^{d}} \xi\right||U|\right) \chi^{2} e^{\xi}+2\left|\tilde{\nabla}_{\mathbb{H}^{d}} \chi\right|^{2} U^{2} e^{\xi} .
\end{aligned}
$$


Thus we obtain

$$
\begin{aligned}
-\left\langle\tilde{\nabla}_{\mathbb{H}^{d}} U, \tilde{\nabla}_{\mathbb{H}^{d}}\left(U \chi^{2} e^{\xi}\right)\right\rangle \leq & \left(-\frac{1}{2}\left|\tilde{\nabla}_{\mathbb{H}^{d}} U\right|^{2}+\left|\tilde{\nabla}_{\mathbb{H}^{d}} U\right|\left|\tilde{\nabla}_{\mathbb{H}^{d}} \xi\right||U|\right) \chi^{2} e^{\xi} \\
& +2\left|\tilde{\nabla}_{\mathbb{H}^{d}} \chi\right|^{2} U^{2} e^{\xi}
\end{aligned}
$$

By (8), (9), (10), (11) and Proposition 4, we have

$$
\begin{aligned}
& {\left[\int_{\mathbb{H}^{d}} U^{2} \chi^{2} e^{\xi} d g\right]_{a}^{b}=\int_{a}^{b} \int_{\mathbb{H}^{d}} \frac{\partial \xi}{\partial s} U^{2} \chi^{2} e^{\xi} d g d s+2 \int_{a}^{b} \int_{\mathbb{H}^{d}} \frac{\partial U}{\partial s} U \chi^{2} e^{\xi} d g d s} \\
& =\int_{a}^{b} \int_{\mathbb{H}^{d}} \frac{\partial \xi}{\partial s} U^{2} \chi^{2} e^{\xi} d g d s+2 \int_{a}^{b} \int_{\mathbb{H}^{d}}\left(\Delta_{\mathbb{H}^{d}} U\right) U \chi^{2} e^{\xi} d g d s \\
& =\int_{a}^{b} \int_{\mathbb{H}^{d}} \frac{\partial \xi}{\partial s} U^{2} \chi^{2} e^{\xi} d g d s-2 \int_{a}^{b} \int_{\mathbb{H}^{d}}\left\langle\tilde{\nabla}_{\mathbb{H}^{d}} U, \tilde{\nabla}_{\mathbb{H}^{d}}\left(U \chi^{2} e^{\xi}\right)\right\rangle d g d s \\
& \leq \int_{a}^{b} \int_{\mathbb{H} d} \frac{\partial \xi}{\partial s} U^{2} \chi^{2} e^{\xi} d g d s \\
& +2 \int_{a}^{b} \int_{\mathbb{H}^{d}}\left\{\left(-\frac{1}{2}\left|\tilde{\nabla}_{\mathbb{H}^{d}} U\right|^{2}+\left|\tilde{\nabla}_{\mathbb{H}^{d}} U\right|\left|\tilde{\nabla}_{\mathbb{H}^{d}} \xi\right||U|\right) \chi^{2} e^{\xi}+2\left|\tilde{\nabla}_{\mathbb{H}^{d}} \chi\right|^{2} U^{2} e^{\xi}\right\} d g d s \\
& \leq \int_{a}^{b} \int_{\mathbb{H}^{d}}\left(-\left|\tilde{\nabla}_{\mathbb{H}^{d}} \xi\right| U^{2}-\left|\tilde{\nabla}_{\mathbb{H}^{d}} U\right|^{2}+2\left|\tilde{\nabla}_{\mathbb{H}^{d}} U\right|\left|\tilde{\nabla}_{\mathbb{H}^{d}} \xi\right||U|\right) \chi^{2} e^{\xi} d g d s \\
& +4 \int_{a}^{b} \int_{\mathbb{H}^{d}}\left|\tilde{\nabla}_{\mathbb{H}^{d}} \chi\right|^{2} U^{2} e^{\xi} d g d s \\
& =-\int_{a}^{b} \int_{\mathbb{H}^{d}}\left(\left|\tilde{\nabla}_{\mathbb{H}^{d}} \xi\right||U|-\left|\tilde{\nabla}_{\mathbb{H}^{d}} U\right|\right)^{2} \chi^{2} e^{\xi} d g d s+4 \int_{a}^{b} \int_{\mathbb{H}^{d}}\left|\tilde{\nabla}_{\mathbb{H}^{d}} \chi\right|^{2} U^{2} e^{\xi} d g d s \\
& \leq 4 \int_{a}^{b} \int_{\mathbb{H}^{d}}\left|\tilde{\nabla}_{\mathbb{H}^{d}} \chi\right|^{2} U^{2} e^{\xi} d g d s .
\end{aligned}
$$

Hence we obtain

$$
\left[\int_{\mathbb{H}^{d}} U^{2} \chi^{2} e^{\xi} d g\right]_{a}^{b} \leq 4 \int_{a}^{b} \int_{\mathbb{H}^{d}}\left|\tilde{\nabla}_{\mathbb{H}^{d}} \chi\right|^{2} U^{2} e^{\xi} d g d s
$$

Since $\left|\tilde{\nabla}_{\mathbb{H}^{d}} \chi\right| \leq \frac{1}{R}$, supp $\left|\tilde{\nabla}_{\mathbb{H}^{d}} \chi\right| \subset \operatorname{supp} \chi=B_{3 R}$ and $\chi \equiv 1$ in $B_{2 R}$, by (12) we obtain

$$
\int_{B_{R}} U_{b}^{2} e^{\xi(g, a)} d g \leq \int_{B_{4 R}} U_{a}^{2} e^{\xi(g, b)} d g+\frac{4}{R^{2}} \int_{a}^{b} \int_{B_{4 R} \backslash B_{2 R}} U^{2} e^{\xi} d g d s .
$$
Since

By Proposition 3, we can set $\sigma(g)=\left(d_{C C}(g)-R\right)_{+}$. Put $l=2 b-a \notin[a, b]$ in (7).

$$
b-a \leq l-s \leq 2(b-a)
$$


for any $s \in[a, b]$, we have

$$
\xi(g, s)=-\frac{\sigma^{2}(g)}{4(l-s)} \leq-\frac{\sigma^{2}(g)}{8(b-a)} \leq 0 .
$$

Hence we can see that

$$
\begin{aligned}
& \xi(g, b)=-\frac{\sigma^{2}(g)}{4(l-b)}=0, \quad g \in B_{R}, \\
& \xi(g, a)=-\frac{\sigma^{2}(g)}{4(l-a)} \leq 0, \quad g \in B_{4 R}
\end{aligned}
$$

and

$$
\xi(g, s) \leq-\frac{\sigma^{2}(g)}{8(b-a)} \leq-\frac{R^{2}}{8(b-a)}, \quad g \in B_{4 R} \backslash B_{2 R} .
$$

Therefore from (13), we obtain the following estimate

$$
\int_{B_{R}} U_{b}^{2} d g \leq \int_{B_{4 R}} U_{a}^{2} d g+\frac{4}{R^{2}} \int_{a}^{b} \int_{B_{4 R} \backslash B_{2 R}} U^{2} \exp \left(-\frac{R^{2}}{8(b-a)}\right) d g d s .
$$

By the assumption of Lemma 1, we have

$$
\int_{a}^{b} \int_{B_{4 R}} U^{2} d g d s \leq e^{f(4 R)} \text { and } \quad b-a \leq \frac{R^{2}}{8 f(4 R)} .
$$

Hence from (14) we obtain

$$
\begin{aligned}
\int_{B_{R}} U_{b}^{2} d g & \leq \int_{B_{4 R}} U_{a}^{2} d g+\frac{4}{R^{2}} \exp \left(-\frac{R^{2}}{8(b-a)}+f(4 R)\right) \\
& \leq \int_{B_{4 R}} U_{a}^{2} d g+\frac{4}{R^{2}} \exp (-f(4 R)+f(4 R)) \\
& =\int_{B_{4 R}} U_{a}^{2} d g+\frac{4}{R^{2}} .
\end{aligned}
$$

This completes the proof of Lemma 1.

ACKNOWLEDGMENT. The author would like to thank the referee for very careful reading of this manuscript and for pointing out several mistakes as well as for the valuable comments and suggestions. 


\section{References}

[ 1 ] O. CAlin, D-C. Chang and P. Greiner, Geometric Analysis in the Heisenberg Group and Its Generalizations, AMS/IP Studies in Advanced Mathematics, 40, American Mathematical Society, Providence, RI, 2007.

[ 2 ] L. CORWIN and F. P. GReENLEAF, Representations of Nilpotent Lie Groups and Their Applications: Volume 1, Part 1, Basic Theory and Examples, Cambridge University Press, Cambridge, 1990.

[ 3 ] B. Franchi, R. SERAPIONi and F. Serra CASSANo, Approximation and imbedding theorem for weighted Sobolev spaces associated with Lipschitz continuous vector fields, Bollettino U. M. I. 7, 11-B (1997), 83117.

[ 4 ] G. B. Folland, Harmonic Analysis in Phase Space, Princeton University Press, Princeton, N. J., 1989.

[ 5 ] N. Garofalo and D. M. NhieU, Lipchitz continuity, global smooth approximations and extension theorems for Sobolev functions in Carnot-Carathéodory spaces, J. Anal. Math. 74 (1998), 67-97.

[ 6 ] A. GRIGOR'YAN, On stochastically complete manifolds, DAN SSSR 290 (1986), no. 3, 534-537 (in Russian).

[ 7 ] A. GRIGOR'YAn, Heat Kernel and Analysis on Manifolds, AMS, International Press, Providence, 2009.

[ 8 ] R. Howe, On the role of the Heisenberg group in harmonic analysis, Bull. Amer. Math. Soc. 3 (1980), 821843.

[ 9 ] Bumsik KIM, Poincaré inequality and the uniqueness of solutions for the heat equation associated with subelliptic diffusion operators, arXiv: 1305.0508v1 [math. AP], 2 May 2013.

[10] S. LI and R. SCHUL, The traveling salesman problem in the Heisenberg group: Upper bounding curvature, Trans. Amer. Math. Soc. 368 (2016), 4585-4620.

[11] T. Matsuzawa, A calculus approach to the hyperfunctions I, Nagoya Math. J. 108 (1987), 53-66.

[12] R. Monti, Some properties of Carnot-Caratheodory balls in the Heisenberg group, Atti Accad. Naz. Lincei Cl. Sci. Fis. Mat. Natur. Rend. Lincei (9) Mat. Appl. 11 (2000), no. 3, 155-167.

[13] Y. OKA, The Schwartz kernel theorem for the tempered distributions in the Heisenberg group, Hokkaido Math. J. 44 (2015), 425-439.

[14] Y. OKA, A characterization of the tempered distributions supported by a regular closed set in the Heisenberg group, Tsukuba J. Math. 39 (2015), no. 1, 97-119.

[15] E. M. SteIn, Harmonic Analysis: Real-Variable Methods, Orthogonality, and Oscillatory Integrals, Princeton University Press, Princeton, N. J., 1993.

[16] S. Thangavelu, An Introduction to the Uncertainty Principle: Hardy's Theorem on Lie Groups, Birkhäuser, Boston, 2004.

[17] A. N. Tikhonov, Uniqueness theorem for the equation of heat conduction, Matem. Sbornik 42 (1935), 199215 (in Russian).

Present Address:

General Education, Department of Creative Engineering,

NATIONAL INSTITUTE OF TECHNOLOGY, KUSHIRO COLLEGE,

2-32-1 OTANOSHIKE-NISHI, KUSHIRO-SHI, HOK KAIDO 084-0916, JAPAN.

e-mail: oka@kushiro-ct.ac.jp 